\title{
Tunable Energy Absorption Characteristics of Architected Honeycombs Enabled via Additive Manufacturing
}

\author{
S. Kumar, ${ }^{* \dagger}{ }^{\dagger}$ J. Ubaid, ${ }^{\dagger}$ R. Abishera, ${ }^{\dagger}$ A. Schiffer, ${ }^{\dagger}$ and V. S. Deshpande ${ }^{\S}$ \\ ${ }^{\dagger}$ Department of Mechanical Engineering, Khalifa University of Science and Technology, Masdar Campus, Masdar City, Abu Dhabi, \\ United Arab Emirates \\ ${ }^{\ddagger}$ Department of Mechanical Engineering, Khalifa University of Science and Technology, Abu Dhabu Campus, Abu Dhabi, United \\ Arab Emirates \\ ${ }^{\S}$ Department of Engineering, University of Cambridge, Trumpington Street, Cambridge CB2 1PZ, United Kingdom
}

Supporting Information

ABSTRACT: Tailoring of material architectures in three-dimensions enabled by additive manufacturing (AM) offers the potential to realize bulk materials with unprecedented properties optimized for location-specific structural and/or functional requirements. Here we report tunable energy absorption characteristics of architected honeycombs enabled via material jetting AM. We realize spatially tailored 3D printed honeycombs (guided by FE studies) by varying the cell wall thickness gradient and evaluate experimentally and numerically the energy absorption characteristics. The measured response of architected honeycombs characterized by local buckling (wrinkling) and progressive failure reveals over $110 \%$ increase in specific energy absorption (SEA) with a concomitant energy absorption efficiency of $65 \%$. Design maps are presented that
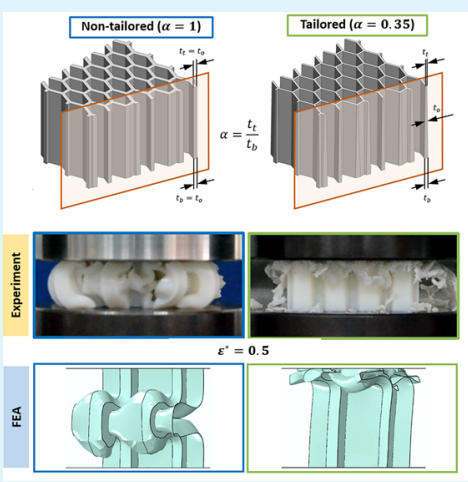

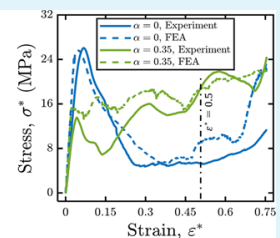

Strain, $\varepsilon^{*}$

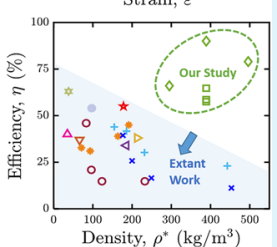
demarcate the regime over which geometric tailoring mitigates deleterious global buckling and collapse. Our analysis indicates that an energy absorption efficiency as high as $90 \%$ can be achieved for architected honeycombs, whereas the efficiency of competing microarchitected metamaterials rarely exceeds $50 \%$. The tailoring strategy introduced here is easily realizable in a broad array of AM techniques, making it a viable candidate for developing practical mechanical metamaterials.

KEYWORDS: additive manufacturing, architected honeycombs, energy absorption, spatial tailoring, cellular materials

\section{INTRODUCTION}

Advances in additive manufacturing (AM) techniques and architectural design of materials have led to the development of high-performance cellular structures for multifarious multifunctional applications. Inspiration for the architectural design of these cellular structures often comes from naturally occurring materials such as cork, sponges, ${ }^{1,2}$ beak and bone of birds, ${ }^{3}$ gecko foot pads, ${ }^{4}$ wood, and plant leaves ${ }^{5-8}$ because of their excellent unique property combinations such as specific stiffness, specific strength, and specific energy absorption ability together with thermal, acoustic, and biological properties. $^{9-15}$ Extensive studies have been carried out on cellular structures because of their potential applications ranging from energy absorbing structures, thermal insulators, and acoustic dampeners to scaffolds for biomedical engineering. $6,9,13,14,16,17$ Properties of cellular materials can be altered to suit the application by independently changing the architectural parameters such as unit-cell configuration and symmetry, ligament size and shape, nodal topology, and the constituent material. ${ }^{18,19}$ The combination of optimized cellular architecture with high-performance constituent materials gives rise to cellular structures with an excellent and unique combination of properties, providing scope for widening the existing property space. ${ }^{17,20}$ Properties of most natural and engineered cellular solids with random and sporadic porosity substantially degrade with a decrease in density because their ligaments predominantly flex under load exhibiting a quadratic or stronger scaling relationship between mechanical properties and relative density. ${ }^{21,22}$ Such size effects observed in the parent material are leveraged by reducing the characteristic structural dimensions for developing a revolutionary class of materials referred to as metamaterials. Metamaterials decouple the conventionally linked properties such as strength and density as they contain micro- and nano-scale architectures arranged in an ordered hierarchy. ${ }^{9,21,23-26}$

Honeycombs are a class of architected cellular materials that combine properties such as lightness, stiffness, strength and high-energy absorbing capabilities that cannot be achieved by

Received: July 24, 2019

Accepted: September 30, 2019

Published: September 30, 2019 
(a)

(b)

(c)

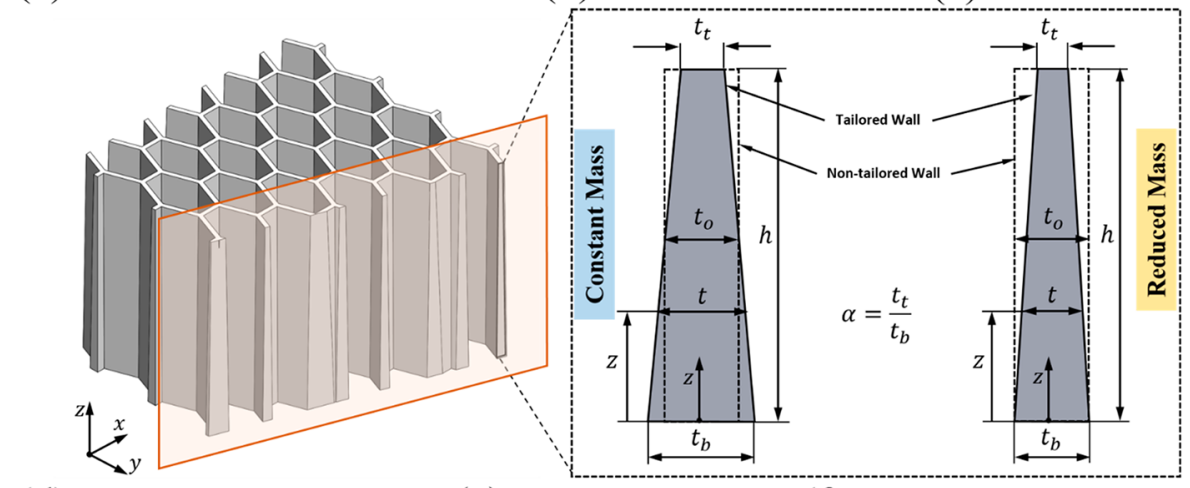

(d)

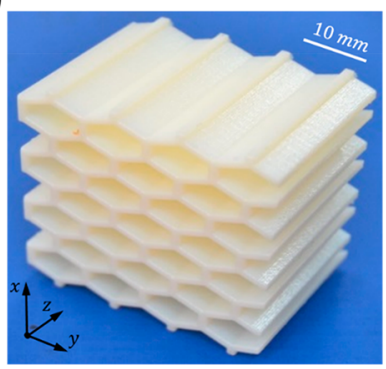

(e)

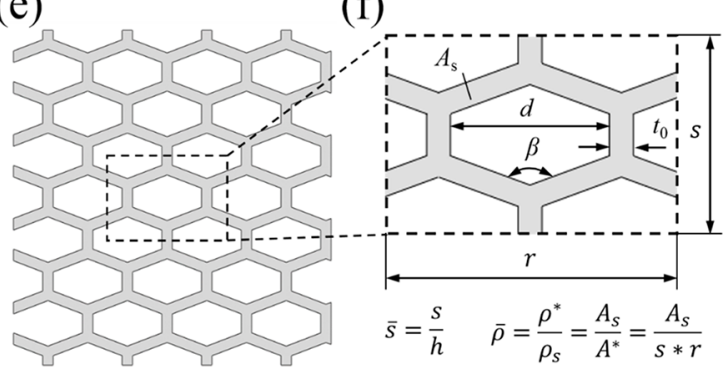

Figure 1. Geometrically tailored additively manufactured irregular hexagonal honeycomb: (a) CAD model. (b, c) Geometrical tailoring schemes for constant mass and reduced mass cases respectively, where $\alpha$ is the gradation parameter. (d) Optical image of 3D printed tailored honeycomb. (e) Irregular honeycomb topology. (f) Geometric details of the irregular honeycomb RVE of size $r$ by $s$; relative density, $\bar{\rho}=\rho * / \rho_{\mathrm{s}}$, and aspect ratio, $\bar{s}=$ $s / h$, where $\rho^{*}$ is the macroscopic density of the cellular material and $\rho_{\mathrm{s}}$ is the density of the solid (basis material). We use $r=20.7 \mathrm{~mm}, s=13.8$ $\mathrm{mm}, h=40 \mathrm{~mm}$, and $t_{0}=1.8 \mathrm{~mm}$.

uniform fully solid materials. They are the most commonly employed two-dimensional bioinspired cellular solids for energy absorbing structures under both crushing and impact conditions. Honeycomb structures have widespread applications in diverse fields ${ }^{27,28}$ because of their high strength-toweight ratio and high stiffness-to-weight ratio ${ }^{29}$ and excellent thermal protection ${ }^{30}$ and superior energy absorption and protective characteristics. ${ }^{31-33}$ Micro- and nano-architected 3D honeycombs are extensively being explored for a multitude of application areas such as energy storage, ${ }^{34}$ reconfigurable electronics ${ }^{35}$ and biomedical. ${ }^{36}$ For instance, a recent study has demonstrated that hierarchically porous $3 \mathrm{D}$ honeycomb-like structures with interconnected porosity can be efficiently used for $\mathrm{CO}_{2}$ capture. ${ }^{37}$ Yet another study has reported biomass derived $3 \mathrm{D}$ honeycomb-like carbon foam for high-performance supercapacitor electrodes. ${ }^{38}$ Another interesting study has utilized crab shell to synthesize honeycomb-like graphitized hierarchically porous carbons for all-solid-state supercapacitors. $^{39}$

In-plane as well as out-of-plane loaded honeycomb structures have been extensively characterized employing theoretical, numerical, and experimental methods. ${ }^{40-46}$ The ever-increasing demand for weight reduction and improved energy absorption has led to improved design and development of lightweight cellular structures with high energy absorption capabilities under extreme loading conditions. Strategies such as introduction of imperfection, corrugation, and hierarchy into the structure have been explored to improve the mechanical performance and mitigate failure characteristics of honeycombs. Imperfections in the honeycomb structure prevent global buckling but promote local buckling, mitigating failure initiation and propagation. ${ }^{47}$ Inspired by the toughening mechanisms observed in hierarchical natural materials such as bamboo, ${ }^{48,49}$ studies have been performed by introducing structural hierarchy into conventional lattice materials to enhance the energy absorption capacity. ${ }^{50-53}$ Incorporation of filler materials such as foams into honeycombs or reinforcing fibers into ligaments is yet another approach to improve the energy absorption performance. ${ }^{54,55}$ Crushing strength and energy absorption characteristics of cellular structures with filler materials and/or cellular composites are greatly enhanced because of the change in buckling behavior of the members of the cellular structure.

Spatial tailoring of architecture and/or material properties $^{15,17,56-62}$ is another emerging key strategy for the development of ultralightweight and damage-tolerant cellular materials. Fabrication of complex tailored cellular structures is challenging, expensive and often impossible via traditional manufacturing techniques. ${ }^{58}$ Advances in AM techniques facilitate fabrication of such sophisticated structures at different length scales with excellent control over the intricate internal architecture. ${ }^{15,19,63,64}$ Modern AM techniques enable fabrication of cellular structures with spatially tailored material properties and geometry. ${ }^{6}$ Furthermore, high-performance computers together with powerful computational tools empower integrated computational materials design through numerical analysis and optimization of these complex structures to enhance the target application. ${ }^{19}$ Studies on cellular materials with spatially varying material properties have shown improvement in energy absorption performance. ${ }^{56,65}$ Geometrically tailored cellular structures exhibit enhanced energy absorption capacity compared to non-tailored structures. ${ }^{31,57,59,66,67}$ Geometric tailoring is usually achieved either by spatially varying the unit cell size or the ligament size. ${ }^{31,58}$ 
As the deformation of honeycomb with higher relative density under out-of-plane compression is characterized by global buckling and collapse, we envision that such deleterious failure mode could be mitigated by spatially tailoring the geometry of the honeycomb. Herein, we explore the energy absorption characteristics of architected honeycombs (irregular hexagonal, re-entrant, and chiral) both experimentally and numerically. Guided by FE studies, geometrically tailored designs were realized for various cell wall thickness gradients via material jetting AM. The energy absorption characteristics, governed by the relative density $\bar{\rho}$, wall thickness gradient parameter $\alpha$, and the aspect ratio of the unit cell $\bar{s}$ of out-ofplane compressive-loaded tailored honeycombs, were measured for different choices of $\alpha$ and found that the tailored honeycombs exhibit an increase in specific energy absorption (SEA) of $110 \%$ for $\bar{\rho}=0.33$ and $\bar{s}=0.35$ with a concomitant increase in energy absorption efficiency of $65 \%$. The damageplasticity FE model (predictions validated by the experimental results) was used to explore the parametric design space and identify architectural parameters $(\bar{\rho}, \alpha$, and $\bar{s})$ that optimize the energy absorption characteristics. FE parametric studies show that the energy absorption characteristics of relatively highdensity honeycombs $(\bar{\rho} \approx 25-35 \%)$ can be substantially improved by employing the geometrical tailoring strategy proposed in this study. Our analysis further reveals that an energy absorption efficiency as high as $90 \%$ can be achieved and that the tailored honeycombs rival most nano- and microarchitected mechanical metamaterials in terms of energy absorption efficiency.

\section{MATERIALS AND METHODS}

2.1. Geometrical Tailoring. The geometrical tailoring of honeycombs is achieved by varying the cell wall thickness along the out-of-plane $(z)$ direction as shown in Figure $1 \mathrm{a}-\mathrm{c}$. Two different tailoring schemes, namely constant mass and reduced mass, are employed in this study. In constant mass tailoring, the mass (and hence the relative density $\bar{\rho}$ ) of the tailored honeycombs is kept the same as that of non-tailored honeycombs. The relative density $(\bar{\rho})$ of the cellular solid is defined as the ratio of the density of the lattice material $\rho^{*}$ to the density of the solid material from which it is made $\rho_{\mathrm{s}}, \bar{\rho}=\frac{\rho^{*}}{\rho_{\mathrm{s}}}$. To maintain same relative density, the material is redistributed by linearly varying the cell wall thickness from the bottom $(z=0)$ at which the wall thickness is $t_{\mathrm{b}}$ to $t_{\mathrm{t}}$ at $z=h$. The wall thickness of the cell at any $z$ for constant mass tailoring is therefore given by

$$
t=t_{\mathrm{b}}\left[1-(1-\alpha) \frac{z}{h}\right] \text { where } t_{\mathrm{b}}=\frac{2 t_{0}}{1+\alpha}
$$

where $\alpha=\frac{t_{\mathrm{t}}}{t_{\mathrm{b}}}$ is the gradation parameter such that $0<\alpha \leq 1, h$ is the height of the honeycomb, and $t_{0}$ is the wall thickness of the nontailored honeycomb. The cell wall thickness at height $z$ for the reduced mass tailoring scheme is given by

$$
t=t_{\mathrm{b}}\left[1-(1-\alpha) \frac{z}{h}\right] \text { where } t_{b}=t_{0}
$$

We introduce another non-dimensional parameter, aspect ratio $\bar{s}=\frac{s}{h}$, where $s$ is the smallest planar size of the unit cell or representative volume element (RVE) (see Figure 1e, f). The three non-dimensional architectural parameters that dictate the macroscopic behavior of the honeycombs are $\bar{\rho}, \alpha$, and $\bar{s}$.

2.2. 3D Printing and Testing of Honeycombs. Polyjet additive manufacturing of photocurable resins is employed to fabricate tailored and non-tailored honeycombs. This AM technique enables fabrication of complex structures with spatially varying geometry and/or materials. Object260 Connex Polyjet 3D printer (Statasys Ltd., USA) is used for the sample fabrication. CAD models of the honeycomb structures were created using Solidworks (Dassault Systems, France). The 3D printer is capable of combining two acrylate-based, UV (ultraviolet) curable resins namely; VeroWhitePlus (VW) and TangoPlus to produce a range of materials with varying properties called digital materials. With this printer, samples can be fabricated with a resolution of $16 \mu \mathrm{m}$ in $z$ - direction and $42 \mu \mathrm{m}$ in the $x$ - and $y$-directions. ${ }^{6-72}$ Here, VW is used to fabricate all the samples. The fabrication is executed in a layer-by-layer fashion concurrently UV-curing each layer of photocurable resin. A support material, SUP705 is used to improve surface finish of the samples and to provide support for overhanging structures. This support material is removed by water jetting. All printing parameters were kept the same for all the samples to avoid any variation of material properties among the samples. Additively manufactured hexagonal honeycomb structure is shown in Figure 1 together with the $\mathrm{CAD}$ model and geometrical details. Together with honeycomb structures, dogbone samples and cylindrical samples were fabricated to evaluate the mechanical behavior of the VW under tension and compression loading, respectively. The stress-strain response under tensile and compressive loading is shown in Figure S1.

Quasi-static compression tests on honeycomb structures and cylindrical compression samples were conducted using an MTS Exceed Series 40 - Universal testing machine with a load cell of 250 $\mathrm{kN}$ having an accuracy of $< \pm 1 \%$ of the reading. An encoder-based position measurement system with a resolution of $0.017 \mu \mathrm{m}$ is used for displacement measurement. Quasi-static tensile tests on the dogbone samples were conducted using a Zwick-Roell universal testing machine with $2.5 \mathrm{kN}$ load cell. The load cell has an accuracy of $< \pm 1 \%$ for the measurement range of 2.5 to $10 \mathrm{~N}$, and $< \pm 0.25 \%$ for the measurement range of 10 to $2500 \mathrm{~N}$ and the displacement was measured with a travel resolution of $0.04 \mu \mathrm{m}$. All the samples were tested in displacement control mode at a strain rate of $0.2 \mathrm{~min}^{-1}$. Every case was printed and tested at least three times to ensure repeatability of the results. Videos of the honeycomb structures under quasi-static compression were captured using a digital camera to examine the crushing behavior of different cases.

2.3. Finite Element Analysis. To simulate the postbuckling response of geometrically tailored and non-tailored honeycombs, we performed FE calculations using the commercial code ABAQUS. Because the measured response of non-tailored irregular honeycomb structure is superior to that of chiral and re-entrant cell topologies (see Figure 7b), only irregular hexagonal honeycombs were considered in the numerical analysis. As shown in Figure le, $\mathrm{f}$, a rectangular unit cell (width $r=20.7 \mathrm{~mm}$, depth $s=13.8 \mathrm{~mm}$ ) of the periodic hexagonal honeycomb structure was modeled and meshed using four-node shell elements with reduced integration (S4RS in ABAQUS), hourglass control, and a total of nine integration points in the through-thickness direction. It was discretized using a uniform mesh size of $l_{\mathrm{e}}=0.25 \mathrm{~mm}$, as shown in Figure S2, and the gradient in the wall thickness of the honeycomb was modeled by adjusting the thickness of each shell element according to an appropriately chosen analytical field. The modeled samples had gradation parameter in the range of $0.2 \leq \alpha \leq 1.0$, average wall thicknesses between $1.35 \mathrm{~mm} \leq$ $t_{0} \leq 2.3 \mathrm{~mm}$, and the sample height was chosen either $h=20 \mathrm{~mm}$ or $40 \mathrm{~mm}$. Periodic boundary conditions were applied on the six edges located parallel to the boundaries of the rectangular unit cell, to mimic the response of an infinite periodic structure. The top and bottom faces of the honeycomb were in contact with rigid analytical surfaces (representing the loading platens of the testing machine), and the top surface was displaced axially at a slow rate to cause quasi-static compression of the honeycomb, whereas the bottom surface was kept fixed, as in the experiments. Note that all contact interactions were modeled using the default normal contact behavior (i.e., "hard" contact) in ABAQUS, whereas a friction coefficient of 0.3 was used to prevent excessive tangential sliding between the rigid surfaces and the FE mesh. The constitutive response of the VW material was modeled using the damage-plasticity model originally developed by Lubliner et 
(a)

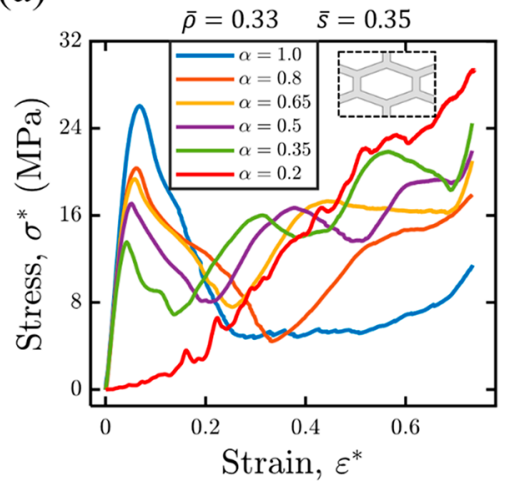

(b)

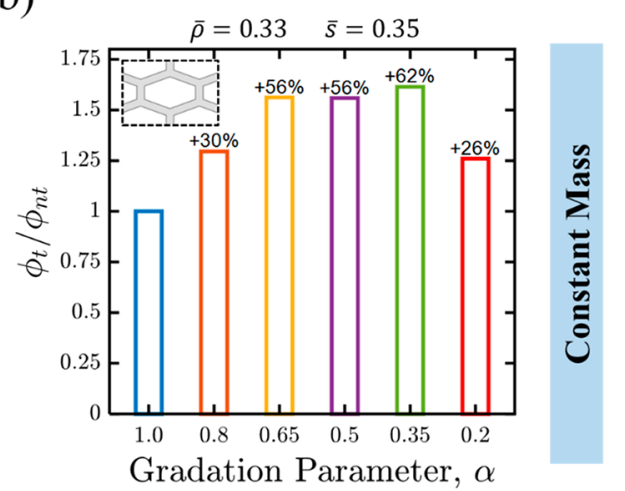

Figure 2. Crushing response and energy absorption characteristics of irregular hexagonal honeycombs for different choices of gradation parameter, $\alpha$ (constant mass tailoring) with relative density $\bar{\rho}=0.33$ and aspect ratio $\bar{s}=0.35$ : (a) stress - strain response and (b) normalized SEA as a function of $\alpha . \phi_{\mathrm{t}}$ is the SEA capacity of tailored honeycombs $(0<\alpha \leq 1)$ and $\phi_{\mathrm{t}}$ is the SEA capacity of nontailored honeycomb $(\alpha=1)$.

al. $^{73}$ for reinforced concrete. The model uses a combined DruckerPrager and Rankine yield surface, which was calibrated using the uniaxial tension and compression stress vs strain data presented in Figure $S 1$ as well as the plasticity parameters defined in Table S2.1. A non-associated flow rule was chosen with the dilation angle set close to zero to mimic the response of a plastically incompressible solid. Material failure in tension was included in the constitutive description by using a bilinear softening curve (also referred to as "tension stiffening curve" in ABAQUS) according to the values listed in Table S2.2. Note that these values were calibrated to match the measured stress vs strain response in uniaxial tension, see Figure S1a. Prior to the onset of plastic deformation, the response of the VW was considered as isotropic and linear elastic with Young's modulus $E_{\mathrm{s}}=$ $1.8 \mathrm{GPa}$, Poisson's ratio $\nu_{\mathrm{s}}=0.41$ and density $\rho_{\mathrm{s}}=1180 \mathrm{~kg} \mathrm{~m}^{-3}$. A constitutive description of the model is given in the Supporting Information S2.

The numerical analysis was carried out in two consecutive steps. First, we performed a buckling analysis in ABAQUS/Standard to identify the dominating buckling modes of each of the honeycomb structures considered. Note that the buckling analysis was performed with the same geometry, mesh, and boundary conditions as described above. Second, a postbuckling analysis was performed by imposing small perturbations (perturbation amplitudes $<t_{0} / 40$ ) on the FE mesh according to the displacement field associated with the first buckling mode of the honeycomb structure. To avoid convergence issues during the analysis, we performed all finite deformation loading calculations in ABAQUS/Explicit with a sufficiently low displacement rate $(500 \mathrm{~mm} / \mathrm{s})$ to eliminate dynamic effects and ensure quasi-static deformation. Buckling and folding processes during the collapse phase of the honeycomb were modeled using the General contact capability in ABAQUS/Explicit with the same normal and tangential contact behavior as described above. The analysis was performed up to a nominal compressive strain of $75 \%$ (as in the experiments), and the obtained force vs displacement profiles were used to determine the stress vs strain responses as well as the specific energy absorption capacities of the honeycombs.

\section{RESULTS AND DISCUSSION}

3.1. Experimental Observations and Measurements. The engineering stress-strain response of irregular hexagonal honeycombs is measured from static out-of-plane compression experiments (see Figure 1a, loading at $z=h$ along $z$ ) performed on $4 \times 4$ cell structures realized via material jetting photopolymer additive manufacturing. The effect of geometrical tailoring (of cell wall) on the energy absorption characteristics of hexagonal honeycombs for different choices of gradation parameter $\alpha$, relative density $\bar{\rho}$ (in reduced mass tailoring scheme) and the aspect ratio $\bar{s}$ (to analyze edge effects) is experimentally evaluated.

3.1.1. Constant Mass Geometrical Tailoring. Figure 2a, b shows the compressive stress-strain response (up to $75 \%$ strain) and the normalized SEA capacity, respectively of geometrically tailored hexagonal honeycombs as a function of $\alpha$ for $\bar{\rho}=0.33$ (constant mass tailoring with $t_{0}=1.8 \mathrm{~mm}$ ) and $\bar{s}$ $=0.35(s=13.8 \mathrm{~mm}$ and $h=40 \mathrm{~mm})$. Note that $\alpha=1$ corresponds to non-tailored honeycomb. In Figure $2 b, \phi_{t}$ is the SEA capacity of tailored honeycombs $(0<\alpha \leq 1)$ and $\phi_{\mathrm{nt}}$ is the SEA capacity of non-tailored honeycomb $(\alpha=1)$. It can be seen that the energy absorption capacity increases with decrease in gradation parameter $\alpha$ such that it attains a maximum (+62\% increase relative to nontailored) for $\alpha=0.35$ and decreases thereafter. With the decrease in $\alpha$ we observe consistent decrease in the peak stress and an improved energy absorption performance (up to $\alpha=0.35$ ). For instance, the peak stress decreased from 25.9 to $13.47 \mathrm{MPa}$ while the SEA increased from 17.26 to $27.87 \mathrm{~J} / \mathrm{g}$ when $\alpha$ is reduced from 1.0 to 0.35 .

The deformation and failure characteristics of geometrically tailored honeycombs that lead to enhanced energy absorption capacity can be explained with the help of Figures 3 and 4 together with Video SV1. The top rows of Figures 3 and 4 show the optical images of deformation maps for non-tailored $(\alpha=1)$ and optimum case of geometrically tailored $(\alpha=0.35)$

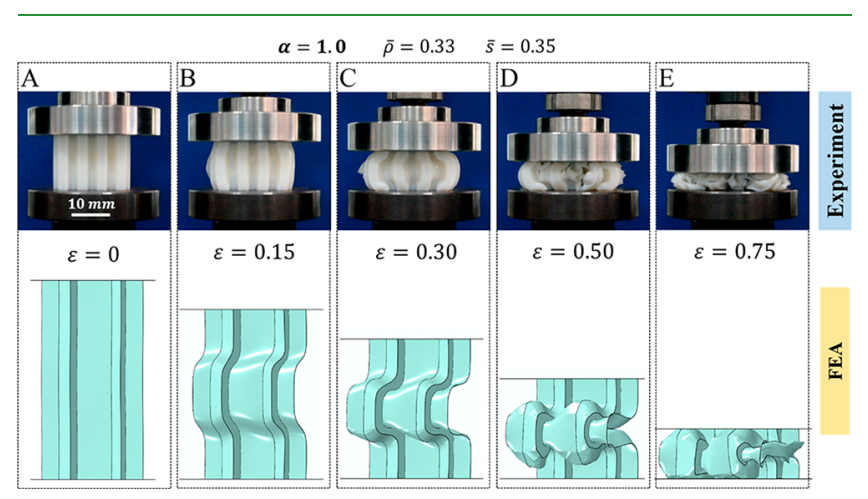

Figure 3. Experiments vs FE predictions: Deformation and failure behavior of nontailored $(\alpha=1)$ irregular hexagonal honeycombs for relative density $\bar{\rho}=0.33$ and aspect ratio $\bar{s}=0.35$. Images $\mathrm{A}-\mathrm{E}$ correspond to compressive strain, $\varepsilon=0,15,30,50$, and $75 \%$, respectively. 


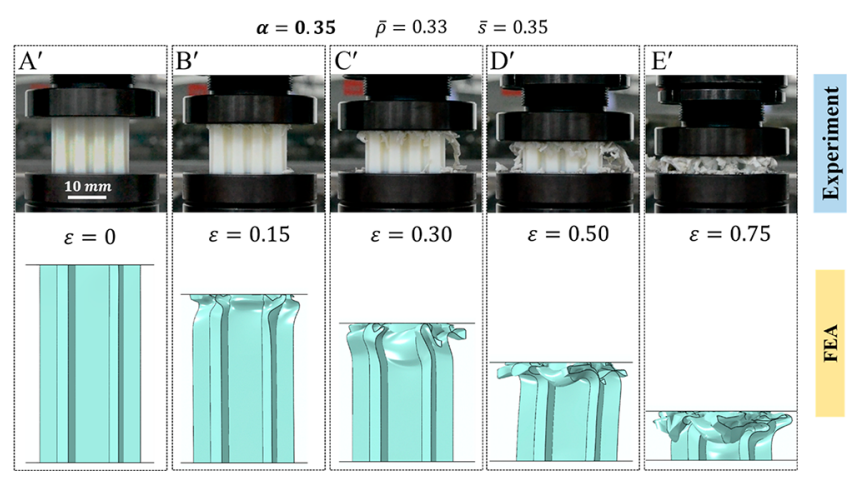

Figure 4. Experiments vs FE predictions: Deformation and failure behavior of tailored $(\alpha=0.35)$ irregular hexagonal honeycombs for relative density $\bar{\rho}=0.33$ and aspect ratio $\bar{s}=0.35 . \mathrm{A}^{\prime}-\mathrm{E}^{\prime}$ show the images at compressive strain, $\varepsilon=0,15,30,50$, and $75 \%$, respectively.

honeycombs respectively at different stages of loading. It can be observed from the images and SV1 that the failure mechanism of non-tailored honeycombs under out-of-plane compression is quite different to that of tailored ones. For nontailored structures $(\alpha=1)$ the following stages are observed: (1) the stress increases linearly to a peak value representing the elastic response, (2) a sharp decrease in the load due to global buckling induced catastrophic failure of the cell walls, (3) a low stress plateau as a result of the fragmentation and splitting of the honeycomb structure, and (4) finally densification denoted by the sharp increase in the stress. In contrast, geometrically tailored structures, postpeak stress, exhibit local buckling and wrinkling progressively (see Figure 2a; incremental load drops that correspond to progressive failure events) starting from the thinnest wall section $(z=40 \mathrm{~mm})$ resulting in a significant increase in the SEA. This progressive failure starting from the thinnest section results in two most desired performance properties of an energy absorbing structure, namely, a reduced initial peak and a sustained crushing plateau. The total energy absorbed and the SEA of hexagonal honeycombs for different choices of $\alpha$ are summarized in Table 1 .

3.1.2. Reduced Mass Geometrical Tailoring. Similar experiments were performed on hexagonal honeycombs for reduced mass tailoring scheme $(\bar{\rho} \propto \alpha$ and $\bar{s}=0.35)$ and the results are presented in Figure S3.1 and summarized in Table S3. In this case, the geometrical tailoring is achieved by reducing the overall mass of the structure. Figure S3.1a, b shows a consistent increase in the SEA with decrease in $\alpha$, exhibiting an optimal increase in the SEA of 38\% for $\alpha=0.35$. Note that the Young's modulus scales as $E \propto \bar{\rho}^{1.03}$ and the yield strength scales as $\sigma_{y} \propto \bar{\rho}^{2.8}$ (see Figure S3.2), indicating that although the modulus is set by cell wall stretching, the strength is governed by local bending of the walls. Although the compressive and energy absorption properties were qualitatively similar to that of constant mass tailoring scheme with similar failure characteristics, quantitative performance improvement is inferior to that of constant mass tailored structures. Nevertheless, we note that the SEA capacity of honeycombs can be enhanced via both geometrical tailoring schemes demonstrated here. Therefore, in the rest of the discussion, we focus on constant mass tailoring scheme unless otherwise stated.

3.1.3. Effect of Cell Topology. We further performed experiments employing constant mass tailoring approach to investigate the effect of cell topology on the performance of
Table 1. Measured Cushing Performance of Irregular Hexagonal Honeycombs for Constant Mass Tailoring ${ }^{a}$

\begin{tabular}{|c|c|c|c|c|}
\hline \multicolumn{5}{|c|}{ Constant Mass Tailoring Scheme } \\
\hline $\begin{array}{c}\text { Cell } \\
\text { Topology }\end{array}$ & $\alpha$ & $\begin{array}{c}\text { Energy Absorbed } \\
(\boldsymbol{J})\end{array}$ & SEA $(J / g)$ & $\begin{array}{c}\text { Efficiency } \\
(\%)\end{array}$ \\
\hline \multirow{6}{*}{$\begin{array}{l}\text { Hexagon } \\
\bar{\rho}=0.33 \\
\bar{s}=0.35\end{array}$} & 1.0 & 786 & 16.4 & 35.8 \\
\hline & 0.8 & $1004(+30 \%)$ & 20.9 & 45.8 \\
\hline & 0.65 & $1220(+56 \%)$ & 25.4 & 55.6 \\
\hline & 0.5 & $1208(+56 \%)$ & 25.2 & 55.1 \\
\hline & 0.35 & $1253(+62 \%)$ & 26.1 & 57.1 \\
\hline & 0.2 & $1042(+26 \%)$ & 21.7 & 47.5 \\
\hline \multirow{6}{*}{$\begin{array}{c}\text { Re-entrant } \\
\bar{\rho}=0.33 \\
\bar{s}=0.35 \\
\end{array}$} & 1.0 & 687 & 14.3 & 31.3 \\
\hline & 0.8 & $1238(+81 \%)$ & 25.8 & 56.4 \\
\hline & 0.65 & $1369(+100 \%)$ & 28.5 & 62.3 \\
\hline & 0.5 & $1396(+108 \%)$ & 29.1 & 63.6 \\
\hline & 0.35 & $1418(+109 \%)$ & 29.6 & 64.7 \\
\hline & 0.2 & $712(+4 \%)$ & 14.9 & 32.5 \\
\hline \multirow{6}{*}{$\begin{array}{c}\text { Chiral } \\
\rho=0.33 \\
\bar{s}=0.35 \\
\end{array}$} & 1.0 & 611 & 12.8 & 27.9 \\
\hline & 0.8 & $979(+60 \%)$ & 20.4 & 44.4 \\
\hline & 0.65 & $1281(+110 \%)$ & 26.7 & 58.2 \\
\hline & 0.5 & $1273(+109 \%)$ & 26.5 & 57.9 \\
\hline & 0.35 & $1289(+111 \%)$ & 26.9 & 58.6 \\
\hline & 0.2 & $922(+49 \%)$ & 19.2 & 42.1 \\
\hline
\end{tabular}

${ }^{a}$ Significant changes in performance vs. non-tailored one are marked in red.

geometrically graded honeycombs. The compressive stressstrain response and the normalized energy absorption characteristics of re-entrant (see Figures 5a, b; $r=20.7 \mathrm{~mm}$, $s=13.8 \mathrm{~mm}$ ) and chiral (see Figures 5a, c; $r=s=13.8 \mathrm{~mm}$ ) honeycombs are presented in Figures $6 \mathrm{a}, \mathrm{b}$ and Figure $6 \mathrm{c}, \mathrm{d}$, respectively. For the same $\bar{\rho}=0.33$ and $\bar{s}=0.35$, tailored reentrant and chiral honeycombs show an optimal increase in energy absorption capacity (for $\alpha=0.35$ as in irregular hexagonal) of 109 and $111 \%$, respectively, relative to their respective non-tailored cases (see Videos SV2 and SV3). The energy absorption characteristics (total energy absorbed and specific energy) of all three cell topologies for different choices of $\alpha$ are summarized in Table 1 with significant increases marked in red. The stress-strain response of optimal structure $(\alpha=0.35)$ and that of the corresponding non-tailored case $(\alpha$ $=1$ ) for three different cell geometries explored are presented in Figures $\mathrm{S} 4 \mathrm{a}-\mathrm{c}$. The normalized SEA capacity (with their respective non-tailored geometry) of all three cell topologies presented in Figure $7 \mathrm{a}$, indicate that the tailored re-entrant $(+109 \%)$ and chiral $(+111 \%)$ honeycombs outperform irregular hexagonal honeycomb $(+62 \%)$ for the same $\bar{\rho}=$ 0.33 and $\bar{s}=0.35$. However, if the performance of all three optimal structures are benchmarked with that of non-tailored hexagonal honeycomb as shown in Figure $7 b$, we notice that all three tailored structures exhibit similar improved performance ( $+62 \%$ for hexagonal, $+74 \%$ for re-entrant and $+60 \%$ for chiral). This is because the non-tailored re-entrant and chiral honeycombs exhibit poor performance as compared to nontailored conventional hexagonal honeycombs. Yet another important performance metric is the energy absorption efficiency of the honeycomb defined as the ratio of the specific energy absorbed by the honeycomb structure, $\phi$ and the specific energy absorbed by the bulk solid material, $\phi_{s}$ when compressed in a uniaxial manner up to a strain of $\varepsilon_{\mathrm{m}}$ given by 
(a)

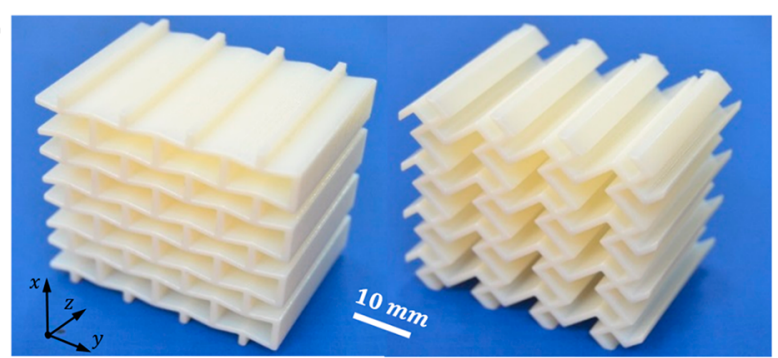

(b)

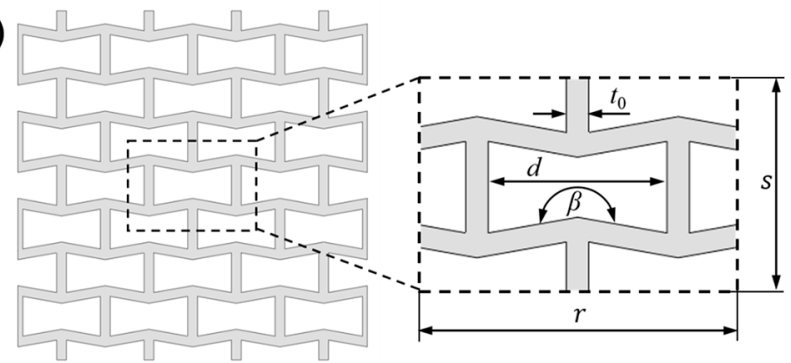

(c)

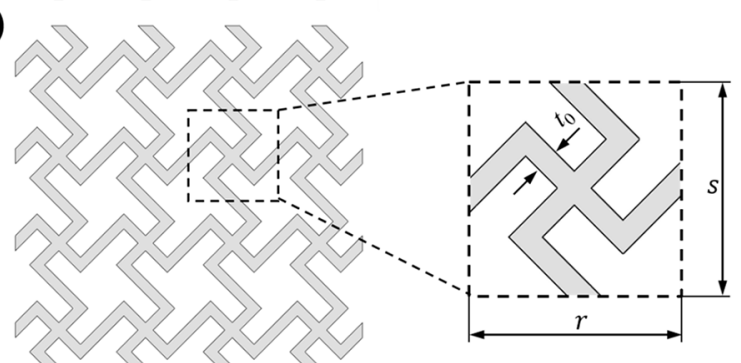

Figure 5. Honeycombs with re-entrant and chiral architectures: (a) Optical images of additively manufactured honeycombs with reentrant and chiral architecture. (b) Re-entrant topology together with geometric details of the RVE of size $r$ by $s$; relative density, $\bar{\rho}=\rho^{*} / \rho_{\mathrm{s}}$ and aspect ratio, $\bar{s}=s / h$, where $\rho^{*}$ is the macroscopic density of the cellular material and $\rho_{\mathrm{s}}$ is the density of the solid (basis material). We use $r=20.7 \mathrm{~mm}, s=13.8 \mathrm{~mm}, h=40 \mathrm{~mm}$ and $t_{0}=1.5 \mathrm{~mm}$. (c) Chiral topology together with geometric details of the chiral honeycomb RVE of size $r$ by $s$. We use $r=13.8 \mathrm{~mm}, s=13.8 \mathrm{~mm}, h=40 \mathrm{~mm}$, and $t_{0}=1.7 \mathrm{~mm}$.

$$
\eta=\frac{\phi}{\phi_{\mathrm{s}}}=\frac{\int_{0}^{\varepsilon_{\mathrm{m}}} \sigma^{*}\left(\varepsilon^{*}\right) \mathrm{d} \varepsilon^{*}}{\int_{0}^{\varepsilon_{\mathrm{m}}} \sigma_{\mathrm{s}}\left(\varepsilon_{\mathrm{s}}\right) \mathrm{d} \varepsilon_{\mathrm{s}}}
$$

where $\sigma_{\mathrm{s}}$ is the macroscopic axial compressive stress experienced by the bulk solid material and $\varepsilon_{\mathrm{s}}$ is the work conjugate of $\sigma_{s}$. Similarly, $\sigma^{*}$ is the axial compressive stress experienced by the honeycomb and $\varepsilon^{*}$ is the work conjugate of $\sigma^{*}$. In here, we calculated the energy density up to a maximum compressive strain $\varepsilon_{\mathrm{m}}=0.75$. Experimentally obtained energy absorption efficiency for all three cell topologies with different choices of $\alpha$ is summarized in Table 1. It is interesting to note that hexagonal honeycomb has the highest efficiency (35.8\%) of all three for nontailored structures $(\alpha=1)$ while the reentrant geometry has the highest efficiency (64.7\%) among tailored cases ( $\alpha=0.35$, see Figure 8 and Figure S4d).

3.1.4. Edge Effects. Edge effects are explored performing experiments on non-tailored $(\alpha=1$ and $\bar{\rho}=0.33)$ and tailored $(\alpha=0.5$ and $\bar{\rho}=0.27)$ hexagonal honeycombs of three different sizes (scaling factor $\mathrm{N}=1(\bar{s}=0.35), 0.75(\bar{s}=0.46)$ and $0.5(\bar{s}=0.69)$, see Figure S5.1) and found that the edge effects are insignificant for both nontailored (see Figure S5.2a, b) and tailored (Figure S5.2c, d) honeycombs, up to $N=0.5$.
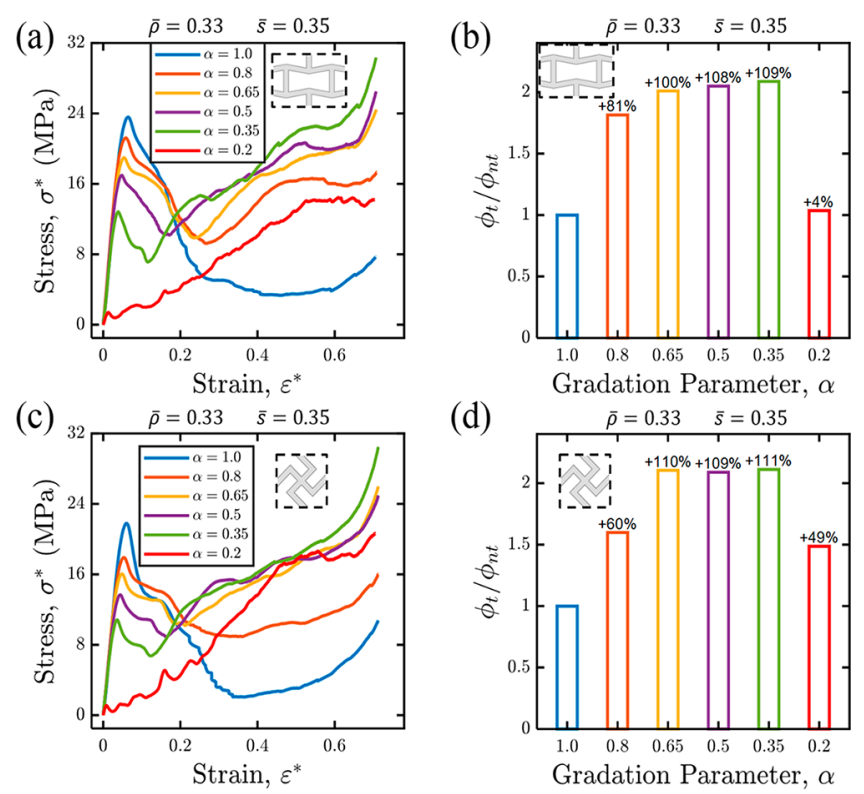

Figure 6. Crushing response and energy absorption characteristics of re-entrant and chiral honeycombs for different choices of gradation parameter, $\alpha$, with relative density $\bar{\rho}=0.33$ and aspect ratio $\bar{s}=0.35$ : (a) Stress-strain response of re-entrant honeycombs for different choices of $\alpha$. (b) Normalized SEA of re-entrant honeycombs as a function of $\alpha$. (c) Stress-strain response of chiral honeycombs for different choices of $\alpha$. (d) Normalized SEA of chiral honeycombs as a function of $\alpha . \phi_{\mathrm{t}}$ is the SEA capacity of tailored honeycombs $(0<\alpha \leq$ $1)$ and $\phi_{\mathrm{nt}}$ is the SEA capacity of nontailored honeycomb $(\alpha=1)$.

3.2. Comparison of Numerical and Experimental Results. To validate our FE model, we proceed to compare experimental observations and measurements to the predictions from the FE model described in Section 2.3. As explained in Section 2.3, only irregular hexagonal honeycombs are considered for this benchmarking. Figures $9 a, b$ show the measured and predicted stress vs strain response of nontailored $(\alpha=1.0)$ and tailored honeycombs $(\alpha=0.35)$ with identical relative density and aspect ratio, $\bar{\rho}=0.33$ and $\bar{s}=$ 0.35 , respectively. The balloons included in these figures indicate selected points on the curves which were earlier used to compare the predicted and observed deformation responses (see Figures 3 and 4). For both tailored (Figure 9b) and nontailored (Figure 9a) cases, the FE predictions are found in good agreement with the measurements, suggesting that our FE model is able to replicate the stress-strain response of tailored honeycombs with good accuracy.

In Figure 10, we show the measured and predicted normalized absorbed energy as a function of the gradation parameter $\alpha$ for the same choices $\bar{\rho}=0.33$ and $\bar{s}=0.35$. Although the FE model slightly overpredicts the measured normalized energy, it is able to reproduce the measured trends and exhibits optimality of energy absorption within the range of $\alpha$ considered here, further confirming the adequacy of our FE model.

In Figure 3, we compare the predicted deformation histories to those observed experimentally (optical images captured at different strain levels up to a maximum strain of 0.75 ) for the non-tailored honeycomb $(\alpha=1.0)$ with $\bar{\rho}=0.33$ and $\bar{s}=0.35$; note that the corresponding stress vs strain response is presented in Figure 9a. From the experimental evidence in Figure 3 (see Video SV1) we observe that the non-tailored 

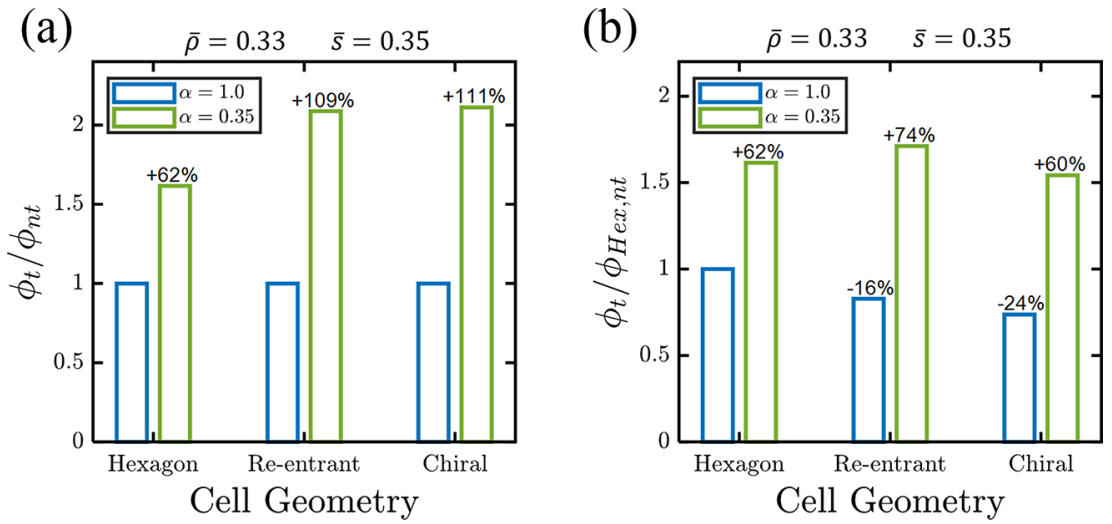

Figure 7. Energy absoption characterisitcs of non-tailored $(\alpha=1)$ and tailored $(\alpha=0.35)$ honeycombs with relative density, $\bar{\rho}=0.33$ and aspect ratio, $\bar{s}=0.35$ : (a) SEA of non-tailored $(\alpha=1)$ and tailored $(\alpha=0.35)$ honeycombs with different architectures normalized with their respective SEA of non-tailored case $\left(\phi_{\mathrm{nt}}\right)$. (b) SEA of honeycombs normalized by SEA of nontailored hexagonal honeycomb $\left(\phi_{\text {Hex,nt }}\right)$.

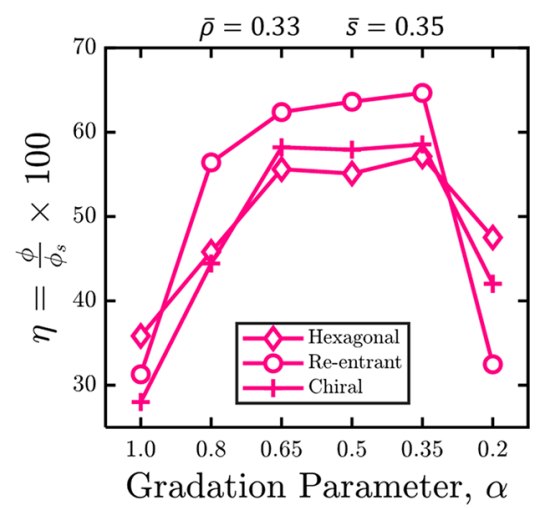

Figure 8. Energy absorption efficiency $\left(\alpha=\phi / \phi_{\mathrm{s}} \times 100\right)$ of nontailored and tailored honeycombs as a function of $\alpha$ for different architectures with relative density $\bar{\rho}=0.33$ and aspect ratio $\bar{s}=0.35$.

honeycomb evinces global buckling and collapse subsequent to the occurrence of the peak load (see Figure 9a), and the corresponding FE predictions confirm this. Similar information is shown in Figure 4 for the tailored case $(\alpha=0.35, \bar{\rho}=0.33, \bar{s}$ $=0.35)$ with the corresponding stress-strain curves shown in Figure $9 \mathrm{~b}$. In contrast to the observation in Figure 3 for the non-tailored structure, the deformation response of the tailored honeycomb with identical relative density and aspect ratio (see Figure 4) is governed by local buckling and folding of the graded cell walls (wrinkling), resulting in a progressive failure

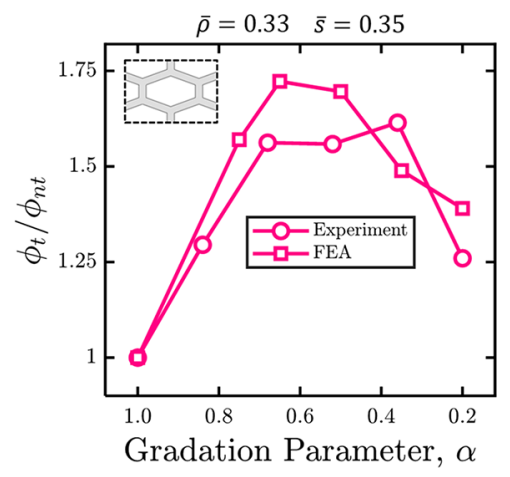

Figure 10. Experiments vs FE predictions of irregular hexagonal honeycombs for relative density $\bar{\rho}=0.33$ and aspect ratio $\bar{s}=0.35$ : Normalized SEA of hexagonal honeycombs as a function of gradation parameter, $\alpha . \phi_{\mathrm{t}}$ is the SEA capacity of tailored honeycombs $(0<\alpha \leq$ $1)$, and $\phi_{\mathrm{nt}}$ is the SEA capacity of nontailored honeycomb $(\alpha=1)$.

mechanism (see Video SV1) and superior energy absorption (see Figures 4, 9b, and 10). Again, the predicted deformation history is found in good agreement with the experimental evidence, affirming that the modeling approach adopted here is adequate to capture the key features of the collapse process associated with geometrically tailored honeycombs.

3.3. Parametric FE Study. In this section, the validated FE model is used to explore the effects of relative density $\bar{\rho}$ and aspect ratio $\bar{s}$ on the response of irregular hexagonal honeycombs with gradation parameter in the range of 0.35
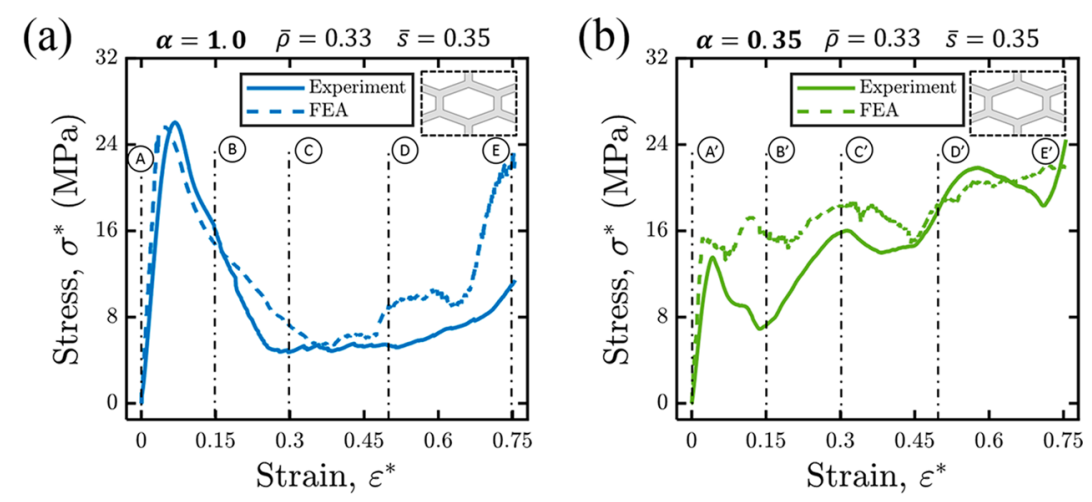

Figure 9. Experiments vs FE predictions of irregular hexagonal honeycombs for relative density $\bar{\rho}=0.33$ and aspect ratio $\bar{s}=0.35$ : (a) stress-strain response of nontailored honeycombs $(\alpha=1)$. (b) Stress-strain response of tailored honeycombs $(\alpha=0.35)$. 

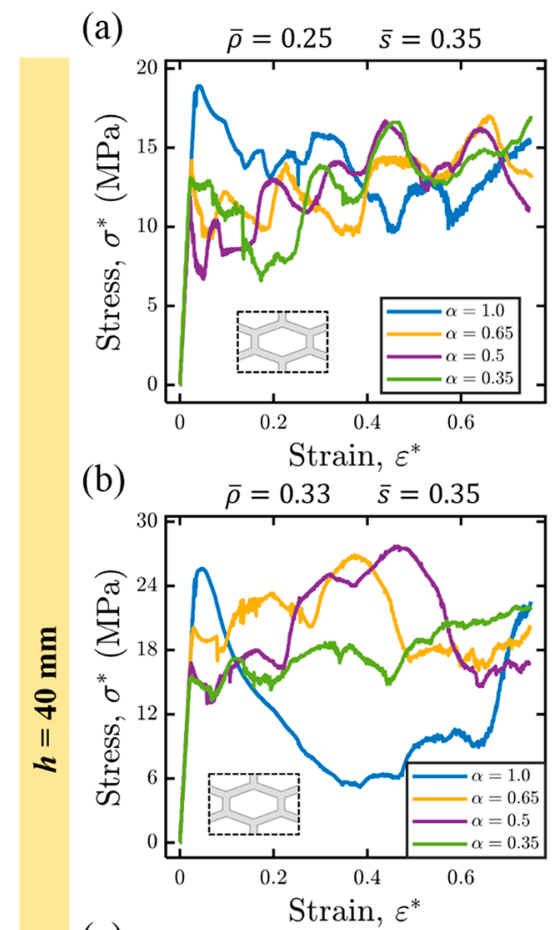

(d)
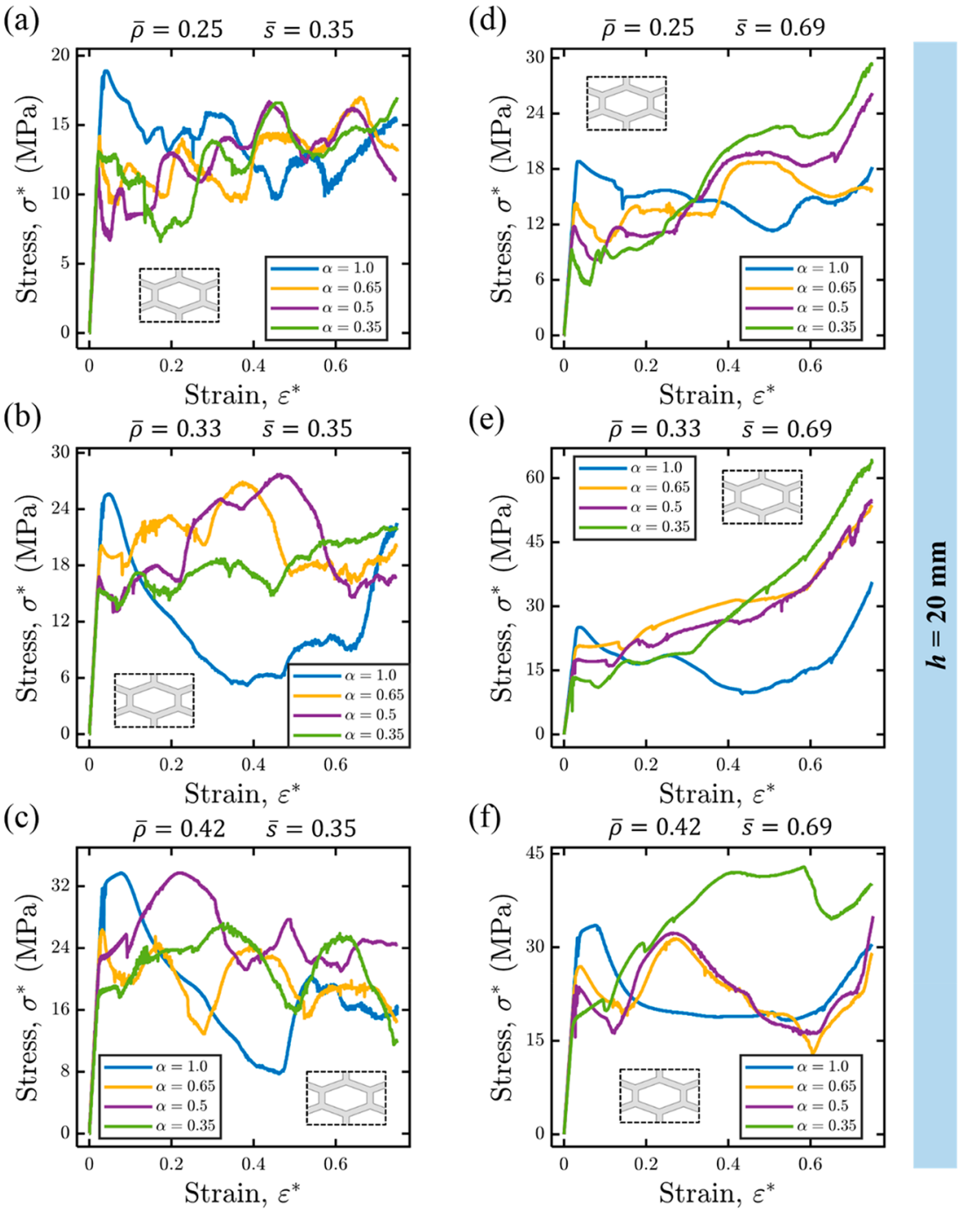

Figure 11. Stress-strain responses (FE predictions) for different choices of $\alpha$ and for different relative densities $\bar{\rho}$ and aspect ratios $\bar{s}$ : (a) $\bar{\rho}=0.25$ and $\bar{s}=0.35$, (b) $\bar{\rho}=0.33$ and $\bar{s}=0.35$, (c) $\bar{\rho}=0.42$ and $\bar{s}=0.35$, (d) $\bar{\rho}=0.25$ and $\bar{s}=0.69$, (e) $\bar{\rho}=0.33$ and $\bar{s}=0.42$, and (f) $\bar{\rho}=0.42$ and $\bar{s}=0.69$.
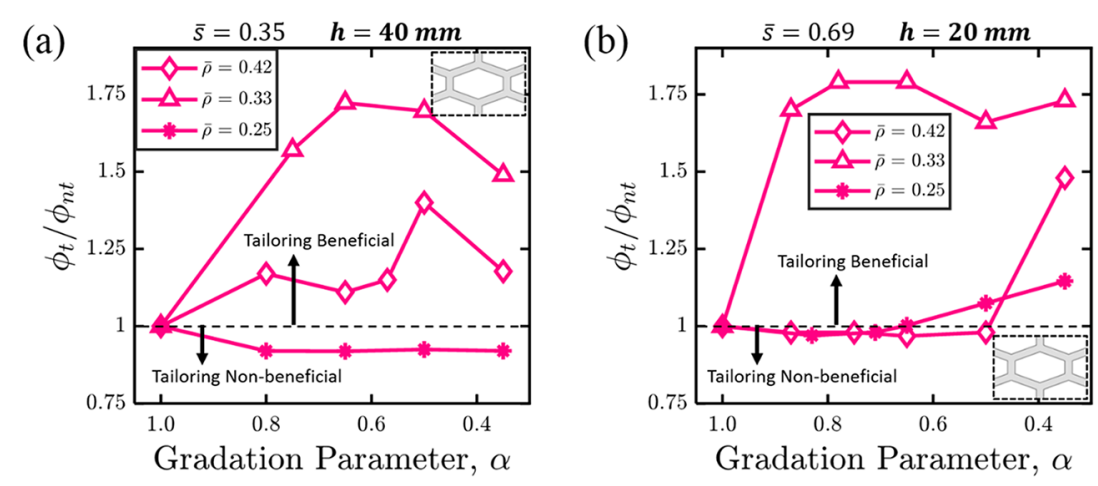

Figure 12. Normalized SEA (FE predictions) of irregular hexagonal honeycombs as a function of gradation parameter, $\alpha$ for different choices of relative density: (a) $\bar{s}=0.35$ (b) $\bar{s}=0.69$.

$\leq \alpha \leq 1.0$. Three different choices of relative density and two choices of aspect ratio were considered, namely $\bar{\rho}=0.25 / 0.33$ / 0.42 and $\bar{s}=0.35 / 0.69$, respectively, giving a total of six different honeycomb geometries. For each of the latter geometries, four FE calculations were performed using $\alpha=$ $1.0 / 0.65 / 0.50 / 0.35$, and the obtained stress vs strain predictions as well as the corresponding normalized absorbed energies (evaluated at a strain of $75 \%$ ) are plotted in Figures 11 and 12 , respectively.

To illustrate the effect of relative density $\bar{\rho}$ for a constant aspect ratio of $\bar{s}=0.35$ (or, $h=40 \mathrm{~mm}$ ), Figures $11 \mathrm{a}-\mathrm{c}$ are now compared. For the case of $\bar{\rho}=0.25$, it is seen from Figure 11a that the stress-strain responses are qualitatively similar for all choices of $\alpha$, showing the non-beneficial effect of geometric 
tailoring on the overall compressive response of the honeycomb. Indeed, Figure 12a shows that for $\bar{\rho}=0.25$, tailoring has deleterious effect, albeit, insignificant, on the SEA. These observations evince that the cell walls of the nontailored honeycomb $(\alpha=1.0)$ do not suddenly collapse (recall Figure 3 ) but progressively fail by local buckling and folding, if the average cell wall thickness (the parameter controlling $\bar{\rho}$ ) is sufficiently small, as illustrated in Figure S6. If such progressive failure is observed for a nontailored honeycomb, our results suggest that no significant benefits in terms of energy absorption capacity would be expected by adopting the concept of geometric tailoring. However, if the relative density is increased to $\bar{\rho}=0.33$, the collapse mechanism of the nontailored honeycomb changes from local to global buckling (see Figure S6) associated with a sudden drop in the predicted stress-strain curve following the first load peak (see Figure $S 6 a)$. Such sudden drop in the load bearing capacity does not occur in the responses of the tailored honeycombs $(\alpha<1.0)$ which exhibit stress fluctuations with smaller amplitudes (see Figure $11 \mathrm{~b}$ showing progressive failure events and efficient material utilization) and a more stable collapse response, associated with local buckling, and this, in turn, gives rise to a substantial increase in absorbed energy, as seen from Figure 12a. Similar trends in the stress-strain responses are observed for the case of $\bar{\rho}=0.42$ (see Figure 11c), however, the normalized absorbed energies associated with these honeycombs are smaller compared to their slightly lighter counterparts with $\bar{\rho}=0.33$, for the entire range of $\alpha$ considered here (see Figure 12a), suggesting that there exists an optimal relative density to maximize the beneficial effect of geometric tailoring. Indeed, guided by the observations from the preliminary FE analyses of nontailored honeycombs (see Figure S6), we chose $\bar{\rho}=0.33$ in our experimental program to explore efficacy of geometric tailoring.

To explore the effect of the unit cell's aspect ratio $\bar{s}$, we now compare the predictions in Figure $11 \mathrm{a}-\mathrm{c}(\bar{s}=0.35)$ to those in Figure $11 \mathrm{~d}-\mathrm{f}(\bar{s}=0.69)$, respectively. For the case of $\bar{\rho}=0.25$ (Figure 11d), the effect of increasing the aspect ratio to $\bar{s}=$ 0.69 (or reducing the height to $h=20 \mathrm{~mm}$ ) is to introduce a considerable degree of hardening in the collapse response of the tailored honeycombs $(\alpha<1.0)$, which becomes more pronounced with decreasing $\alpha$ and results in a slight increase in normalized absorbed energy, as seen from Figure $12 \mathrm{~b}$. Similar trends are observed for the cases $\bar{\rho}=0.33$ (Figure 11e) and $\bar{\rho}=$ 0.42 (Figure 11f), though, yet again, we observe maximum benefit for $\bar{\rho}=0.33$ as a result of geometric tailoring (see Figure $12 \mathrm{~b}$ ). Nevertheless, for the choice of $\bar{\rho}=0.42$, major differences between the stress-strain responses of tailored and nontailored honeycombs are detected only for the case of $\alpha=$ 0.35 , resulting in a sudden increase in normalized absorbed energy, as seen from Figure 12b. Again, this sudden change in energy absorption can be attributed to the transition from a sudden or catastrophic collapse mechanism to one dominated by local buckling and progressive failure, which occurs at a lower value of $\alpha$ due to the low height and large relative density of the latter honeycombs. The design maps presented in Figure 12 clearly demarcate the regime over which tailoring is beneficial. Key predictions from FE parametric studies are summarized in Table S7. Figure 13 shows energy efficiency versus density map for architected honeycombs compared to most common energy absorbing materials. ${ }^{74-77}$ Most commercially available honeycombs have relative densities less than $5 \%$, warranting the use of relatively high-density

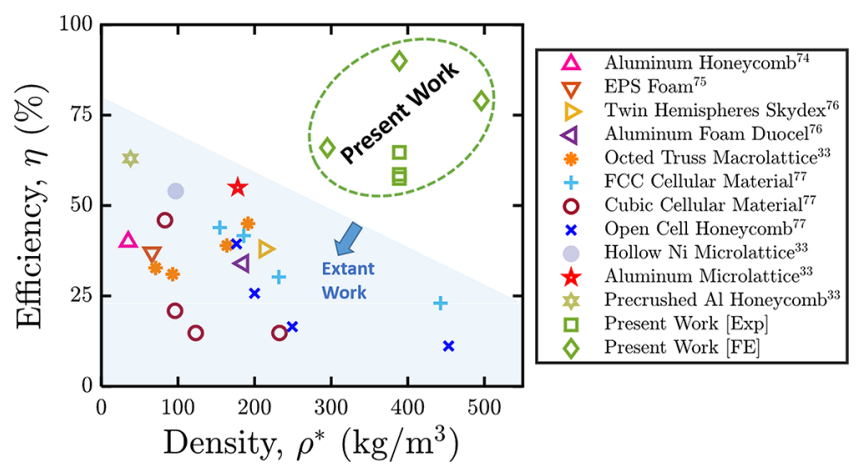

Figure 13. Energy absorption efficiency versus density map for architected honeycombs compared to most common energy absorbing materials.

geometrically tailored honeycombs, which exhibit optimal energy absorption characteristics. We further observe that an optimal choice of relative density $(\bar{\rho}=0.33)$ makes tailored honeycombs tenable, yielding a maximum energy absorption efficiency of $90 \%$ for $\bar{s}=0.69$ at $\alpha=0.65$.

\section{CONCLUSIONS}

In this study, enhanced energy absorption characteristics of geometrically tailored honeycombs (irregular hexagonal, reentrant, and chiral) enabled via material jetting additive manufacturing were experimentally demonstrated and numerically evaluated. The geometrically tailored designs were realized by varying the cell wall thickness along the out-ofplane direction. The energy absorption characteristics, governed by the relative density $\bar{\rho}$, wall thickness gradient parameter $\alpha$, and the aspect ratio of the unit cell, $\bar{s}$ of out-ofplane compressive-loaded irregular hexagonal, re-entrant and chiral honeycombs were measured for different choices of $\alpha$ and found that all three tailored honeycombs exhibit an optimal SEA at $\alpha=0.35$ (irregular hexagonal: $+62 \%$, reentrant: $+109 \%$ and chiral: $+111 \%)$ for the same $\bar{\rho}=0.33$ and $\bar{s}=0.35$. However, we notice that all three tailored honeycombs exhibit similar improved SEA (+62\% for hexagonal, $+74 \%$ for re-entrant and $+60 \%$ for chiral) with respect to nontailored hexagonal honeycomb. Experimentally obtained energy absorption efficiency of all three cell topologies for different choices of $\alpha$ indicates that the reentrant geometry has the highest efficiency of $64.7 \%$ (hexagonal: $57.1 \%$ and chiral: $58.6 \%$ ) for $\alpha=0.35$. The damage-plasticity FE model benchmarked with experimental results was used to explore the parametric design space (varying $\alpha, \bar{\rho}$, and $\bar{s}$ ) and identify architectural parameters that optimize the energy absorption characteristics. The design maps presented clearly demarcate the regime over which tailoring mitigates global buckling and collapse. We show that for a choice of relative density $\bar{\rho}=0.33$, an energy absorption efficiency as high as $90 \%$ can be achieved for our architected honeycombs and note that the SEA efficiency of extant microarchitected metamaterials seldom exhibits 50\%. Exceptional performance enhancement due to effective material utilization and progressive wrinkling reaffirms the potential of our architected honeycombs for diverse applications. This study has shown that tailoring such honeycombs opens the possibility for them to be designed to fulfill specific material requirements, such as prescribed stiffness, strength, and energy absorption by controlling the collapse modes. However, 
applications of such architected honeycombs go well-beyond traditional mechanical applications. As an example, consider energy recovery from the heel of shoe comprising a honeycomb core. Approximately $60 \mathrm{~W}$ of power are available from heel strikes during a brisk walk ( $68 \mathrm{~kg}$ person, 2 steps $/ \mathrm{s}$, heel moving $50 \mathrm{~mm}$ ). While this level of energy extraction is not feasible, a sole comprising a "flexible" sandwich stave comprising a piezo honeycomb core and electroded face sheets can exploit the high pressures exerted on the heel. Structural stability and energy absorption capability become critical requirements for such a honeycomb core and this study represents a step toward the mechanical optimization of such a honeycomb core. The convergence of emerging nanoscale AM techniques and the ability to design nano- and microarchitected hierarchical structures with tightly controlled architecture will enable the realization of new class of materials with unprecedented structural and/or functional properties.

\section{ASSOCIATED CONTENT}

\section{S Supporting Information}

The Supporting Information is available free of charge on the ACS Publications website at DOI: 10.1021/acsami.9b12880.

Material characterization of additively manufactured materials, Finite Element model and constitutive description of the damage-plasticity model, preliminary numerical study on energy absorption characteristics of nontailored hexagonal honeycombs, experimental results of irregular hexagonal, re-entrant and chiral honeycombs for reduced mass tailoring scheme, effect of cell topology and edge effects on measured crushing characteristics of hexagonal honeycombs (PDF)

Video SV1, synchronized load-displacement response and deformation map of tailored vs. non-tailored irregular hexagonal honeycombs for $\bar{\rho}=0.33$ (AVI)

Video SV2, synchronized load-displacement response and deformation map of tailored vs. non-tailored reentrant honeycombs for $\bar{\rho}=0.33$ (AVI)

Video SV3, synchronized load-displacement response and deformation map of tailored vs. non-tailored chiral hexagonal honeycombs for $\bar{\rho}=0.33$ (AVI)

\section{AUTHOR INFORMATION}

\section{Corresponding Author}

*Email: s.kumar@eng.oxon.org (S.K.).

ORCID $\odot$

\section{S. Kumar: 0000-0002-3386-8012}

\section{Author Contributions}

S.K. conceived the idea. J.U. and R.A. performed 3D printing and experiments. A.S. carried out Finite Element simulations. S.K. and V.S.D. guided the project. All authors discussed the results. S.K., J.U., A.S., and V.S.D. contributed to manuscript preparation.

\section{Notes}

The authors declare no competing financial interest.

\section{ACKNOWLEDGMENTS}

We gratefully acknowledge financial support from the ADNOC under Award No: Ex2016-000010. S.K. also thanks Khalifa University for its support towards his research leave (summer 2018) at the University of Cambridge with the financial support from ADNOC.

\section{REFERENCES}

(1) Aizenberg, J.; Weaver, J. C.; Thanawala, M. S.; Sundar, V. C.; Morse, D. E.; Fratzl, P. Skeleton of Euplectella sp.: structural hierarchy from the nanoscale to the macroscale. Science 2005, 309 (5732), 275-278.

(2) Yin, S.; Wang, H.; Li, J.; Ritchie, R. O.; Xu, J. Light but Tough Bio-inherited Materials: Luffa Sponge based Nickel-plated Composites. Journal of the mechanical behavior of biomedical materials 2019, 94, 10.

(3) Fratzl, P.; Weinkamer, R. Nature's hierarchical materials. Prog. Mater. Sci. 2007, 52 (8), 1263-1334.

(4) Yao, H.; Gao, H. Mechanics of robust and releasable adhesion in biology: Bottom-up designed hierarchical structures of gecko. J. Mech. Phys. Solids 2006, 54 (6), 1120-1146.

(5) Gibson, L. J.; Ashby, M. F. Cellular Solids: Structure and Properties; Cambridge University Press: 1999.

(6) Schaedler, T. A.; Carter, W. B. Architected cellular materials. Annu. Rev. Mater. Res. 2016, 46, 187-210.

(7) Gibson, L. J.; Ashby, M. F.; Harley, B. A. Cellular Materials in Nature and Medicine; Cambridge University Press: 2010.

(8) Wegst, U. G.; Bai, H.; Saiz, E.; Tomsia, A. P.; Ritchie, R. O. Bioinspired structural materials. Nat. Mater. 2015, 14 (1), 23.

(9) Yeo, S. J.; Oh, M. J.; Yoo, P. J. Structurally Controlled Cellular Architectures for High-Performance Ultra-Lightweight Materials. Adv. Mater. 2018, 31 (34), 1803670.

(10) Mueller, J.; Raney, J. R.; Shea, K.; Lewis, J. A. Architected Lattices with High Stiffness and Toughness via Multicore-Shell 3D Printing. Adv. Mater. 2018, 30, 1705001.

(11) Wadley, H. N. Multifunctional periodic cellular metals. Philos. Trans. R. Soc., A 2006, 364 (1838), 31-68.

(12) Si, Y.; Yu, J.; Tang, X.; Ge, J.; Ding, B. Ultralight nanofibreassembled cellular aerogels with superelasticity and multifunctionality. Nat. Commun. 2014, 5, 5802.

(13) Sui, N.; Yan, X.; Huang, T.-Y.; Xu, J.; Yuan, F.-G.; Jing, Y. A lightweight yet sound-proof honeycomb acoustic metamaterial. Appl. Phys. Lett. 2015, 106 (17), 171905.

(14) Hollister, S. J. Porous scaffold design for tissue engineering. Nat. Mater. 2005, 4 (7), 518.

(15) Compton, B. G.; Lewis, J. A. 3D-printing of lightweight cellular composites. Adv. Mater. 2014, 26, 5930-5935.

(16) Fleck, N. A. a.; Deshpande, V. S. S.; Ashby, M. F. F. Microarchitectured materials: past, present and future. Proc. R. Soc. London, Ser. A 2010, 466, 2495.

(17) Tancogne-Dejean, T.; Spierings, A. B.; Mohr, D. Additivelymanufactured metallic micro-lattice materials for high specific energy absorption under static and dynamic loading. Acta Mater. 2016, 116, 14-28.

(18) Pham, M.-S.; Liu, C.; Todd, I.; Lertthanasarn, J. Damagetolerant architected materials inspired by crystal microstructure. Nature 2019, 565 (7739), 305.

(19) Schaedler, T. A.; Jacobsen, A. J.; Carter, W. B. Toward lighter, stiffer materials. Science 2013, 341 (6151), 1181-1182.

(20) Zhang, Z.; Zhang, Y.-W.; Gao, H. On optimal hierarchy of loadbearing biological materials. Proc. R. Soc. London, Ser. B 2011, 278 (1705), 519-525.

(21) Zheng, X.; Lee, H.; Weisgraber, T. H.; Shusteff, M.; DeOtte, J.; Duoss, E. B.; Kuntz, J. D.; Biener, M. M.; Ge, Q.; Jackson, J. A.; et al. Ultralight, ultrastiff mechanical metamaterials. Science 2014, 344 (6190), 1373-1377.

(22) Patole, S. P.; Reddy, S. K.; Schiffer, A.; Askar, K.; Prusty, B. G.; Kumar, S. Piezoresistive and Mechanical Characteristics of Graphene Foam Nanocomposites. ACS Applied Nano Materials 2019, 2 (3), $1402-1411$.

(23) Schaedler, T. A.; Jacobsen, A. J.; Torrents, A.; Sorensen, A. E.; Lian, J.; Greer, J. R.; Valdevit, L.; Carter, W. B. Ultralight Metallic Microlattices. Science 2011, 334 (6058), 962-965.

(24) Mohsenizadeh, M.; Gasbarri, F.; Munther, M.; Beheshti, A.; Davami, K. Additively-manufactured lightweight Metamaterials for energy absorption. Mater. Des. 2018, 139, 521-530. 
(25) Greer, J. R.; Park, J. Additive Manufacturing of Nano- and Microarchitected Materials. Nano Lett. 2018, 18 (4), 2187-2188.

(26) Sajadi, S. M.; Owuor, P. S.; Schara, S.; Woellner, C. F.; Rodrigues, V.; Vajtai, R.; Lou, J.; Galvão, D. S.; Tiwary, C. S.; Ajayan, P. M. Multiscale Geometric Design Principles Applied to 3D Printed Schwarzites. Adv. Mater. 2018, 30 (1), 1704820.

(27) Vaziri, A.; Hutchinson, J. W. Metal sandwich plates subject to intense air shocks. Int. J. Solids Struct. 2007, 44 (6), 2021-2035.

(28) Tejavibulya, N.; Youssef, J.; Bao, B.; Ferruccio, T.-M.; Morgan, J. R. Directed self-assembly of large scaffold-free multi-cellular honeycomb structures. Biofabrication 2011, 3 (3), 034110.

(29) Ashby, M. F., Gibson, L. J. Cellular Solids: Structure, Properties and Applications Cork; Press Syndicate of the University of Cambridge: Cambridge, UK (1997): 175-231.

(30) Lu, T.; Chen, C. Thermal transport and fire retardance properties of cellular aluminium alloys. Acta Mater. 1999, 47 (5), 1469-1485.

(31) Ajdari, A.; Nayeb-Hashemi, H.; Vaziri, A. Dynamic crushing and energy absorption of regular, irregular and functionally graded cellular structures. Int. J. Solids Struct. 2011, 48 (3-4), 506-516.

(32) Sun, G.; Jiang, H.; Fang, J.; Li, G.; Li, Q. Crashworthiness of vertex based hierarchical honeycombs in out-of-plane impact. Mater. Des. 2016, 110, 705-719.

(33) Schaedler, T. A.; Ro, C. J.; Sorensen, A. E.; Eckel, Z.; Yang, S. S.; Carter, W. B.; Jacobsen, A. J. Designing Metallic Microlattices for Energy Absorber Applications. Adv. Eng. Mater. 2014, 16 (3), 276283.

(34) Wu, F.; Srot, V.; Chen, S.; Lorger, S.; van Aken, P. A.; Maier, J.; Yu, Y. 3D Honeycomb Architecture Enables a High-Rate and LongLife Iron (III) Fluoride-Lithium Battery. Adv. Mater. 2019, 0 (0), 1905146.

(35) Damdam, A.; Qaisar, N.; Hussain, M. Honeycomb-serpentine silicon platform for reconfigurable electronics. Appl. Phys. Lett. 2019, 115 (11), 112105.

(36) Leonhardt, E. E.; Kang, N.; Hamad, M. A.; Wooley, K. L.; Elsabahy, M. Absorbable hemostatic hydrogels comprising composites of sacrificial templates and honeycomb-like nanofibrous mats of chitosan. Nat. Commun. 2019, 10 (1), 2307.

(37) Shi, W.; Wang, R.; Liu, H.; Chang, B.; Yang, B.; Zhang, Z. Biowaste-derived 3D honeycomb-like $\mathrm{N}$ and $\mathrm{S}$ dual-doped hierarchically porous carbons for high-efficient CO 2 capture. RSC Adv. 2019, 9 (40), 23241-23253.

(38) Huang, J.; Wu, J.; Dai, F.; Li, C. M. 3D honeycomb-like carbon foam synthesized with biomass buckwheat flour for high-performance supercapacitor electrodes. Chem. Commun. 2019, 55 (62), 91689171.

(39) Shi, W.; Chang, B.; Yin, H.; Zhang, S.; Yang, B.; Dong, X. Crab shell-derived honeycomb-like graphitized hierarchically porous carbons for satisfactory rate performance of all-solid-state supercapacitors. Sustainable Energy \& Fuels 2019, 3 (5), 1201-1214.

(40) Mc Farland, R. Hexagonal cell structures under post-buckling axial load. AIAA J. 1963, 1 (6), 1380-1385.

(41) Wierzbicki, T. Crushing analysis of metal honeycombs. International Journal of Impact Engineering 1983, 1 (2), 157-174.

(42) Baker, W.; Togami, T.; Weydert, J. Static and dynamic properties of high-density metal honeycombs. International Journal of Impact Engineering 1998, 21 (3), 149-163.

(43) Ruan, D.; Lu, G.; Wang, B.; Yu, T. X. In-plane dynamic crushing of honeycombs - a finite element study. International Journal of Impact Engineering 2003, 28 (2), 161-182.

(44) Yamashita, M.; Gotoh, M. Impact behavior of honeycomb structures with various cell specifications-numerical simulation and experiment. International Journal of Impact Engineering 2005, 32 (14), 618-630.

(45) Xu, S.; Beynon, J. H.; Ruan, D.; Lu, G. Experimental study of the out-of-plane dynamic compression of hexagonal honeycombs. Compos. Struct. 2012, 94 (8), 2326-2336.

(46) Xiang, Y.; Yu, T.; Yang, L. Comparative analysis of energy absorption capacity of polygonal tubes, multi-cell tubes and honeycombs by utilizing key performance indicators. Mater. Des. 2016, 89, 689-696.

(47) Lee, S.; Hahn, C.; Rhee, M.; Oh, J.-E. Effect of triggering on the energy absorption capacity of axially compressed aluminum tubes. Mater. Eng. 1999, 20 (1), 31-40.

(48) Lakes, R. Materials with structural hierarchy. Nature 1993, 361 (6412), 511.

(49) Habibi, M. K.; Lu, Y. Crack propagation in bamboo's hierarchical cellular structure. Sci. Rep. 2015, 4, 5598.

(50) Chen, Y.; Jia, Z.; Wang, L. Hierarchical honeycomb lattice metamaterials with improved thermal resistance and mechanical properties. Compos. Struct. 2016, 152, 395-402.

(51) Mousanezhad, D.; Babaee, S.; Ebrahimi, H.; Ghosh, R.; Hamouda, A. S.; Bertoldi, K.; Vaziri, A. Hierarchical honeycomb auxetic metamaterials. Sci. Rep. 2016, 5, 18306.

(52) Si, Y.; Yu, J.; Tang, X.; Ge, J.; Ding, B. Ultralight nanofibreassembled cellular aerogels with superelasticity and multifunctionality. Nat. Commun. 2014, 5, 5802.

(53) Ajdari, A.; Jahromi, B. H.; Papadopoulos, J.; Nayeb-Hashemi, H.; Vaziri, A. Hierarchical honeycombs with tailorable properties. Int. J. Solids Struct. 2012, 49 (11), 1413-1419.

(54) Hanssen, A. G.; Langseth, M.; Hopperstad, O. S. Static and dynamic crushing of circular aluminium extrusions with aluminium foam filler. International Journal of Impact Engineering 2000, 24 (5), 475-507.

(55) Chen, W.; Wierzbicki, T. Relative merits of single-cell, multicell and foam-filled thin-walled structures in energy absorption. ThinWalled Structures 2001, 39 (4), 287-306.

(56) Yu, B.; Han, B.; Su, P.-B.; Ni, C.-Y.; Zhang, Q.-C.; Lu, T. J. Graded square honeycomb as sandwich core for enhanced mechanical performance. Mater. Des. 2016, 89, 642-652.

(57) Duan, S.; Tao, Y.; Lei, H.; Wen, W.; Liang, J.; Fang, D. Enhanced out-of-plane compressive strength and energy absorption of $3 \mathrm{D}$ printed square and hexagonal honeycombs with variable-thickness cell edges. Extreme Mechanics Letters 2018, 18, 9-18.

(58) Bates, S. R.; Farrow, I. R.; Trask, R. S. Compressive behaviour of $3 \mathrm{D}$ printed thermoplastic polyurethane honeycombs with graded densities. Mater. Des. 2019, 162, 130-142.

(59) Maskery, I.; Aremu, A.; Parry, L.; Wildman, R.; Tuck, C.; Ashcroft, I. Effective design and simulation of surface-based lattice structures featuring volume fraction and cell type grading. Mater. Des. 2018, 155, 220-232.

(60) Yang, L.; Mertens, R.; Ferrucci, M.; Yan, C.; Shi, Y.; Yang, S. Continuous graded Gyroid cellular structures fabricated by selective laser melting: Design, manufacturing and mechanical properties. Mater. Des. 2019, 162, 394-404.

(61) Panesar, A.; Abdi, M.; Hickman, D.; Ashcroft, I. Strategies for functionally graded lattice structures derived using topology optimization for Additive Manufacturing. Additive Manufacturing 2018, 19, 81-94.

(62) Xiao, Z.; Yang, Y.; Xiao, R.; Bai, Y.; Song, C.; Wang, D. Evaluation of topology-optimized lattice structures manufactured via selective laser melting. Mater. Des. 2018, 143, 27-37.

(63) Tancogne-Dejean, T.; Diamantopoulou, M.; Gorji, M. B.; Bonatti, C.; Mohr, D. 3D Plate-Lattices: An Emerging Class of LowDensity Metamaterial Exhibiting Optimal Isotropic Stiffness. Adv. Mater. 2018, 30 (45), 1803334.

(64) Li, X.; Zhang, J. M.; Yi, X.; Huang, Z.; Lv, P.; Duan, H. Multimaterial Microfluidic 3D Printing of Textured Composites with Liquid Inclusions. Advanced Science 2019, 6 (3), 1800730.

(65) Shen, C.; Lu, G.; Yu, T. Dynamic behavior of graded honeycombs-a finite element study. Compos. Struct. 2013, 98, 282293.

(66) Al-Saedi, D. S.; Masood, S.; Faizan-Ur-Rab, M.; Alomarah, A.; Ponnusamy, P. Mechanical properties and energy absorption capability of functionally graded F2BCC lattice fabricated by SLM. Mater. Des. 2018, 144, 32-44.

(67) Andrew, J. J.; Ubaid, J.; Hafeez, F.; Schiffer, A.; Kumar, S. Impact performance enhancement of honeycombs through additive 
manufacturing-enabled geometrical tailoring. International Journal of Impact Engineering 2019, 134, 103360.

(68) Ubaid, J.; Wardle, B. L.; Kumar, S. Strength and Performance Enhancement of Multilayers by Spatial Tailoring of Adherend Compliance and Morphology via Multimaterial Jetting Additive Manufacturing. Sci. Rep. 2018, 8 (1), 13592.

(69) Khan, M. A.; Kumar, S.; Cantwell, W. J. Additively manufactured cylindrical systems with stiffness-tailored interface: Modeling and experiments. Int. J. Solids Struct. 2018, 152-153, 7184.

(70) Khan, M.; Kumar, S. Performance enhancement of tubular multilayers via compliance-tailoring: $3 \mathrm{D}$ printing, testing and modeling. Int. J. Mech. Sci. 2018, 140, 93-108.

(71) Kumar, S.; Wardle, B. L.; Arif, M. F.; Ubaid, J. Stress Reduction of 3D Printed Compliance-Tailored Multilayers. Adv. Eng. Mater. 2017, 20 (1), 1700883.

(72) Kumar, S.; Wardle, B. L.; Arif, M. F. Strength and Performance Enhancement of Bonded Joints by Spatial Tailoring of Adhesive Compliance via 3D Printing. ACS Appl. Mater. Interfaces 2017, 9, 884.

(73) Lubliner, J.; Oliver, J.; Oller, S.; Oñate, E. A plastic-damage model for concrete. Int. J. Solids Struct. 1989, 25 (3), 299-326.

(74) Liu, Y.; Schaedler, T. A.; Chen, X. Dynamic energy absorption characteristics of hollow microlattice structures. Mech. Mater. 2014, 77, $1-13$.

(75) Caserta, G. D.; Iannucci, L.; Galvanetto, U. Shock absorption performance of a motorbike helmet with honeycomb reinforced liner. Compos. Struct. 2011, 93 (11), 2748-2759.

(76) De Sousa, R. A.; Gonçalves, D.; Coelho, R.; Teixeira-Dias, F. Assessing the effectiveness of a natural cellular material used as safety padding material in motorcycle helmets. Simulation 2012, 88 (5), $580-591$.

(77) Mieszala, M.; Hasegawa, M.; Guillonneau, G.; Bauer, J.; Raghavan, R.; Frantz, C.; Kraft, O.; Mischler, S.; Michler, J.; Philippe, L. Micromechanics of amorphous metal/polymer hybrid structures with 3D cellular architectures: size effects, buckling behavior, and energy absorption capability. Small 2017, 13 (8), 1602514. 